\title{
An e-Education Platform for Teaching-Learning Processes that are Difficult to Visualize
}

\author{
Jorge López ${ }^{1}$, Narciso Cerpa ${ }^{2}$ and Yussef Farran ${ }^{1}$ \\ ${ }^{1}$ Universidad de Concepción, Chile ${ }^{2}$ Universidad de Talca, Chile
}

\begin{abstract}
We present a teaching-learning platform for e-education that was developed to facilitate the bi-directional communication between instructor and learner when teaching-learning the behavior of non-visible processes. This platform also facilitates the educational evaluation of the conceptual and practical understanding of the learner. We discuss cognitive load theory within the context of e-education and its influence on the design and development of an educational platform of this type. We describe the use of this teaching-learning platform in an educational model without the presence of the instructor, and as a complementary material to the courses. We also discuss the experience with this platform when used in networking and operating systems courses. We describe those characteristics of this platform that simplify and facilitate the communication between learner and instructor. We also describe the current implementation of our e-educational platform and discuss the evaluation of our platform by students of networking and operating systems courses in a distance learning program.
\end{abstract}

Keywords: e-Learning, e-Teaching, Cognitive Load, Visualization, Animation

\section{INTRODUCTION}

The advent of e-education is making distance learning a reality, and education institutions are now using a variety of asynchronous and/or synchronous platforms for achieving their purposes. However, most of these platforms do not consider the cognitive aspects of learning. There is a large body of evidence suggesting that many traditional instructional techniques may unnecessarily overload limited working memory and impede learning (Cooper et al.,1987; Chandler et al.,1991; Chandler et el.,1992; Kalyuga et al.,1998;2000; Kalyuga, 2000; Pass et al.,1994; Sweller, 1988;1989; $1991 ; 1993 ; 1994)$. In this case, the phrase "traditional instructional

\footnotetext{
The original version of this chapter was revised: The copyright line was incorrect. This has been corrected. The Erratum to this chapter is available at DOI: 10.1007/978-0-387-35617-4_48 
techniques" refers to the way the materials are presented rather than the media used. When the teaching materials need to be presented through electronic media, the way the materials are organized and presented plays an important role (Cerpa et al., 1996). Therefore, in this research, we make use of cognitive load theory to design and implement our e-education platform.

Cognitive load theory provides a framework for the design and development of effective teaching materials by taking into account the limitations and strengths of the human information processing architecture (Chandler et al., 1991; Sweller,1988; Sweller et al.,1994). This theory suggests that the limitations of the human memory system have severe implications for the presentation of instructional materials. The limitations imposed by working memory, and its heavy load when processing information to be stored in or retrieved from long-term memory, make necessary the use of cognitive constructs such as schemas to reduce working memory load. The cognitive processes that modify schemas may be categorized into three types: accretion; tuning; and restructuring (Rumelhart,1984; Rumelhart et al., 1981). Accretion is adding new information to existing schemas increasing their flexibility. Tuning is the process of improving a schema to achieve its goals more efficiently. Schema refinement is attained by constant practice and appropriate feedback. Unlike accretion and tuning, restructuring entails the creation of new schemas, and is relevant to learning, because it permits humans to deal with novel problems (Gick et al.,1983; Holyoak,1985). The presence of schemas for a specific domain during intellectual activities is to some degree, a synonym of expertise, since these are essential for quickly identifying relevant features in problems, categorizing problems, and making analogies with already solved problems.

According to cognitive load theory, instructional materials usually impose two different and independent sources of cognitive load (Sweller, 1994). Intrinsic cognitive load, which is determined by the complexity of the instructional material itself (e.g. mental effort involved in understanding and learning the use of registers in the processing of instructions in computers) (Halford et al., 1994). On the other hand, the extraneous cognitive load is imposed solely by the way the instructional material is structured and presented. Extraneous cognitive load places unnecessary and heavy demands on working memory, hindering the acquisition of schemas, their automation, and hence learning (Chandler et al.,1991;1992; Sweller, 1993;1994; Sweller et al., 1994). An instructional method may use different presentation formats: visual (e.g. text, pictures, diagrams, animation, etc.), auditory (e.g. sound) or both, and each of these may impose an extraneous cognitive load. Cognitive load theory suggests that the elimination or reduction of extraneous cognitive load imposed by the structure of instructional materials results in an improvement in learning and problem solving (Sweller, 1994). 
The intrinsic cognitive load is generated by the degree of interactivity between learning elements (Sweller, 1993; 1994; Sweller et al., 1994). Consider students who must learn a second language. The language vocabulary may take a long time to learn because of the large number of elements involved, but each word can be learned in isolation. We can consider each word to be an element, therefore, the intrinsic cognitive load associated with learning this material is relatively low. In this case there is virtually zero or very low interactivity between the elements. High element interactivity tasks occur when the nature of the task is such that the elements must be assimilated simultaneously or processed concurrently in order to understand the material (Halford et al.,1986; Maybery et al.,1986). For example, a second language also requires learning the syntax of the language, and in this case the elements involved cannot be learned in isolation because they heavily interact with each other. As a consequence, tasks such these are high in element interactivity (Halford et al., 1998).

It needs to be emphasized that what constitutes an element depends entirely on previous learning. For someone who is experienced in a language, all of the elements in the syntax of a language have been combined into a schema that acts as a single element. For an expert, that schema will not impose a heavy working memory load. For a novice learning a second language for the first time, a schema incorporating the syntax of the language has not been acquired and each of the constituents must be treated as individual elements held in working memory. As was discussed above, acquisition of new knowledge triggers restructuring of existing schemas and new higher order schemas are created. Novices must acquire lower-order schemas before they can treat the constituent's learning elements as a unit, and be able to learn more difficult materials, acquiring higher order schemas.

Cognitive load theory research has aimed to improve instructional methods by promulgating instructional techniques and designs which facilitate schema acquisition and automation. There have been numerous studies applying cognitive load theory, and the results of these studies have uncovered some pitfalls associated with conventional instructional methods as well as generating new approaches to correct and improve these methods. Some of these cognitive load findings, usually called cognitive load "effects", are worked-example, split-attention, redundancy, and modality. Cognitive load theory places emphasis in the use of worked-examples during learning, to facilitate the learner's understanding of problem structure by presenting the solution, state by state, and identifying the operators, which allow modification of problem states. Although worked-examples have been proven to be more effective than conventional problem solving, cognitive load suggests that under some conditions, worked-examples may be ineffective. Consider a student who must assimilate instructional material from a worked-example consisting of a diagram and text, with neither the 
diagram nor text being intelligible in isolation. Both can become intelligible only after they have been mentally integrated. This is usually called the splitattention effect. Another example of an extraneous cognitive load is the redundancy effect. It occurs when different sources of information that can be understood in isolation are both included in instructional materials resulting in redundancy. While information processing models of learning have emphasized the limitations of working memory, there is evidence (Baddely, 1992; Pavio, 1990) that under some conditions, an expansion of working memory may be achieved. Some portion of working memory is dedicated to attending to visual information only and other portion is dedicated to attending to aural information only. Therefore, rather than attempting to reduce cognitive load, we could virtually "expand" working memory using a mixed mode instructional format (i.e. modality effect) to facilitate learning. However, the value of multiple representations of information has been questioned, because there are conditions when the addition of an audio explanation to visual instructions has negative rather than positive or neutral effects (Hegarty, 1999; Najjar, 1996; Tergan, 1997). Those conditions occur when processing an auditory supplement is likely to impose an excessive working memory load.

On the other hand, the human brain uses the visual and auditory senses within others to associate ideas that once understood can be communicated if required. To achieve this association of ideas, sometimes it must translate images to spoken language and vice versa. In general, we do not always use both, the visual and auditory senses for the communication of ideas as we do in the mental processes. This is because the human being has the facility to use the spoken communication language, which is portable. The visual communication requires the use of external elements that we must learn (drawing, painting). The visual communication skills that we can have depend on the innate skills of each individual. Although we can continue developing these skills, the visual dynamics makes very difficult any bimedial kind of communication. However, the multimedia technology offers a platform that facilitates the solution to this problem.

Our aim is to improve the teaching-learning process, placing emphasis on the bi-directional communication, because this seems to be the only way to ensure that the learner has understood the concepts and acquired the skills we gave to her/him. It is quite usual to test the process of knowledge acquisition using the spoken and written language supported by diagrams to help explanation. Therefore, if the teaching process is supported by multimedia technology, and the evaluation is undertaken without this facility, we have a communication unbalance between teaching and evaluation.

While e-education encourages the use of multimedia, the networking infrastructure and the equipment usually available at the student site suggests the use of media that integrated is educationally effective and do not produce 
overload in the network. As was mentioned above, audio must be used in well-defined conditions, and as we know the use of video produces a heavy use of resources. An alternative visual presentation format is animation, which facilitates the representation of dynamics materials, which could be difficult to teach other way. Animation is just a sophisticated way of presenting graphical and textual information over a period of time. Hence, the materials presented in animations must be cognitively integrated to reduce cognitive load and consequently facilitate learning. The teaching of many computer science topics may be facilitated with the appropriate use of animations (i.e. computer architectures, algorithms, networking, programming, etc). This discipline requires the use of selected workedexamples by the instructor, and learning by doing at the student side. In this case, interactivity is an essential aspect of e-education. Therefore, it is extremely important to provide a platform that facilitates the development of animated worked-examples by the instructor in a diversity of computer science topics (but not restricted to). In the same way, we need to provide the means for interaction with the student, who must be able to follow the worked-examples, play with them and create modified versions of these examples, and develop creativity by generating her/his own structures (i.e. architectures, networks, etc). Although there exist many studies on the use of different modalities and instructional formats, little work has been done on the use of animations according to cognitive load theory. Our objective is to test the cognitive load effects when using animations, and to provide a model for bi-directional interaction between instructor and learner based on animations and cognitive load theory.

Research using single modality visual instructions (Kalyuga et al., 1998;2000; Kalyuga, 2000) indicated that low-knowledge students (i.e. novices) benefited from additional text based information included with diagrams. High knowledge students (i.e. with some experience) showed a preference for an instructional package, which consisted of diagrams only. This suggests that the level of difficulty (i.e. interactivity) depends on the learners' background knowledge, and this has a strong influence on the materials presented (i.e. diagram, text, both). Little work has been done to provide a solution to the learners' background knowledge problem. In our research as part of the interactive model, we pursue to provide generic mechanisms for detecting the level of expertise of the learner, and to adapt the presentation format to this level.

\section{OBJECTIVES}

The general objective of this project is the design, development and evaluation of an e-education platform to support the teaching-learning process that facilitates the info-graphical communication of non-visible 
dynamic processes. We aim to use the same platform and media to evaluate the knowledge acquisition process and its product. Our specific objectives are to:

- build a cognitively based teaching-learning hyper-medial platform to edit and display info-graphical animations

- test the cognitive load effects when using animations, and to provide a model for bi-directional interaction between instructor and learner based on animations and cognitive load theory.

- develop a methodology to evaluate the knowledge acquired by the learner, using the same cognitively based hyper-medial platform

- provide generic mechanisms for detecting the level of expertise of the learner, and to adapt the presentation format to this level.

- analyze the effect of using this teaching-learning platform

\section{TEACHING-LEARNING PLATFORM}

We must keep in mind that the learners must no require special skills to create and manipulate images when using the platform. Therefore, the visual language must be simple and intuitive. However, the teacher must describe the specific info-graphical alphabet before teaching and evaluating the learner. The basic requirements for the teaching-learning platform are that it must:

- be a tool to visualize the dynamic behavior of systems

- allow the experimentation with a language proposed by the teacher of each course

- be appropriate for teaching with and without the presence of the teacher

- be used to build explanations interactively in classes

- be flexible to model and visualize processes with the most appropriate self-explained elements (images, text)

- allow the sequential visualization of processes that reflect the dynamic evolution of the described phenomena

- facilitate the setting and assimilation of animated worked-examples

- provide mechanisms for the forward and backward visualization of processes

- use the minimal number of basic graphical objects in the editing of the animation elements, to simplify this activity

- provide a visual language to facilitate the editing of animations by teachers and students with no need of special skills

- be cognitively-based, and easy to use

Based on these requisites, our model present the following features to the instructor and learner: 
- The instructor can offer an image (info-graphical template), which has transparent areas, and is present in all the animation frames. This template is an intermediate level of depth with respect to the user within each frame.

- The info-graphical language basic code is determined by the chosen template

- The template can be edited with any external graphic tool available to the instructor

- The platform editor component allows the creation of each animation frame through a set of simple geometric objects (line, circle, rectangle), which are placed in a layer behind the transparent zone of the template (lower layer). Therefore, the transparent zone will prevail in the final image.

- The user may also write explanations in form of text in front of the template, using the platform editor

- If the learner has a new proposal for the template, she/he can edit the same simple figures in a higher layer

Each animation frame has three components: graphical frame; mixed (graphical frame with balloons that include explanation text); and a frame with a detailed explanation. The visualization of this set of animation frames has three levels:

- Graphical level : displays all the frames at a constant rate (1 frame / sec)

- Mixed level (balloons with text) : displays the graphical frames plus the explanation text, and its display takes a time proportional to the length of the text to allow its reading

- Help level: displays a conceptual explanation of the current frame (it can be activated by pressing a button).

The animations are categorized in such a way that in the higher layers we use the info-graphical language. In the lower layers we require a higher level of detail, and we make prevail the image. The idea is to substitute a set of frames for an equivalent unique frame, which combines the image with a meaningful text (info-graphical language). There is a previous mental association between the representative frame and the set of frames represented. The issue here is to produce this association in the mental process (Corry, 1998) of the learner who receives the info-graphical message. The idea is to follow an order in which we use the animations going from lower to higher levels. The advantages of applying this order are to:

- significantly reduce the effort required to build the animated infographical explanations

- encourage a textual description of the learnt materials by the learner

- facilitate the evaluation by using higher level animations 
Our approach can be described as the following recursive algorithm:

An image is equivalent to thousand words

A word is equivalent to thousand images

An info-graphical language is equivalent to words plus images

In other words, we must not use many images if we can use a few words, and we must not write many words if we can use a few images.

The instructor may present an animated worked-example and challenge the learner to build an alternative or complementary animation. The instructor may also challenge the learner to textually describe the animation process, and to modify the animation according to her/his interpretation. The feedback from the learner will allow the classification of her/him within the spectrum of background knowledge from novice to expert. This classification is essential for presenting the learner with the appropriate animations to facilitate learning

Our e-education hyper-medial platform can be used in different teachinglearning processes (non-conventional, conventional). For example, as part of an e-education platform for distance learning without the instructor's presence. In this case, the instructor may receive textual explanations through the interactive environment. These explanations allow her/him to modify the animations according to interpretations and understandings. The platform will also automatically determine the experience of the learner and will tailor the animations to her/his experience. The same platform permits the educational evaluation of the learnt materials. The platform may also be used in laboratory situations, with workstations, where the instructor shows animations to the learners, and challenges them to build new alternative and/or complementary animations. This environment is also appropriate to evaluate the learner.

Another environment in which we use this teaching-learning platform is the conventional classroom, but with the use of a data-show and a computer to support the explanations of the instructor. In this case, the platform is used to demonstrate the processes through animations, and to build minianimations replacing the whiteboard and pen.

\section{SOFTWARE PROTOTYPE ARCHITECTURE}

The characteristics of our current software prototype are the followings:

- The communication is through an interactive tool that operates over the world wide web

- The prototype was built using the Java language to ensure portability and the integration to any other e-education platform

- The animation of static components as proposed by the instructor (graphical templates), is added to the process animation through the 
platform editor. These templates may be edited in any graphic design tool capable to generate a GIF format.

- The animation of dynamic components must be created through the platform editor

- The prototype provide options to visualize the animations step-by-step in a controlled fashion, or automatically

- Each animation consists of an image template and a serializable Java object that contains frames of simple figures

- The animation frames are images generated immediately before displaying, and combining the frames of simple figures with the template

- Both, the template and the frame of objects are transported through a network, from and to a storage server

- The communication with the server is achieved using Remote Method Invocation (RMI) Java

Summarizing, our software prototype is a system for editing and displaying animations through a web browser, and satisfies the requirements above.

\section{E-EDUCATION PLATFORM EVALUATION}

The initial prototype of our platform was evaluated by students of networking and operating systems courses. The platform was used in a classroom with data projector for demonstrations, and interactive animation building to substitute the whiteboard. In the operating systems course we had 35 students and the platform was used for approximately 4 hours spread in several sessions. This was complemented by the use of overhead transparencies when needed. We taught the following topics:

- Execution of kernel interruptions

- Commutation of processor's operation mode: User-Supervisor

We obtained the following results:

- It facilitated the explanation of dynamic processes, since the platform allowed interactive modifications by the teacher after receiving input from the students. This is usually more difficult to achieve when using a whiteboard and pen

- The teacher built new explanations in the classroom that were saved and published for further revision from the students

The platform was also used in a distance learning course without the presence of the instructor. In this case the instructor asked the students for textual explanations and improved the animations based on the explanations received. In the distance learning situation, we had the following activities with the students

1. Editing animation (instructor) 
2. Visualize animation (student)

3. Use text to describe the visualization (student)

4. Validation of described text (instructor)

5. If the text validation is not satisfactory, the instructor improves the animation and we go back to step 1

6. Stop loop

In the networking courses we had 45 students (Diploma in Informatics (10 students); and Computer Engineering (35 students) and taught the following topics:

- Visualization of data transmission in an Ethernet with hub and switch without collisions

- Visualization of data transmission in an Ethernet with hub and switch with collisions

- Visualization of packet transmission in networks with hub, switch and routers

- Visualization of the generation and consumption of headers in the protocol of the different layers in a basic network

The students could interact with the instructor through the animations in the e-education platform. The students provided feedback (i.e. text) to the instructor regarding the animations, and the instructor improved the animations based on the students' feedback. This is possible since the infographical template was formulated by an interdisciplinary team and uses a simple visual language that is quite easy to learn. The reproduction of an animation with modifications, does not include modifications to the template, but to the animation itself as it is permitted by the language. The visual language is easy to use, since it was thought as the communication means for the students to respond questions. At the end of the diploma program, the students had to qualitatively evaluate the course providing comments. The module with the best evaluation was networking, and according to the students, the info-graphical animations were relevant to the success of the module.

Another use of our e-education platform was in laboratory conditions, where the instructor shows and explains an animation, challenging the students to build a new complementary animation. In the networking course for Computer Engineering we evaluated the students avoiding to have a negative impact in the final mark of the students. We asked the students to set an animation to represent the sending of the agreement message when receiving a protocol unit. We offered a bonus of $3 \%$ in the final mark for those students who responded correctly in a period of 5 minutes, $2 \%$ in 10 minutes, and $1 \%$ in 15 minutes. We had 10 students that responded in a period of 5 minutes, 12 in 10 minutes, and the rest responded between 10 and 15 minutes. All the students built a correct animation using the infographical template (visual language). The followings are the advantages of 
this teaching-learning platform as observed by the students and the instructor.

General advantages:

- Facilitates visualization of non-visible processes

- Explanations may be minimized regarding frames, cognitive load, and bandwidth

- Reduces need of taking notes of dynamic processes

- Allows the learner to communicate using the same explanation language

- Presents alternatives for visualization

- Uses the Internet as the basic platform

- Facilitates the use of alternative templates Without the presence of the instructor, the learner can:

- Have access to a set of integrated info-graphical explanations of a specific topic

- Activate alternative aspects of the info-graphical explanation (text, image) according to needs

- Follow step-by-step the animation frames

- Create alternative explanations to allow the refinement of the animation by the instructor. This alternative explanations may be stored in the platform administered by the instructor

- Be evaluated using the same explanation language. This produces a balance between teaching and learning, since the instructor can evaluate knowledge in situations that it cannot be assessed by traditional evaluations methods (e.g. paper-based written exams)

- Study the explanations in conjunction with notes, bibliography and other learning materials

- Store the explanation materials in her/his own computer as complementary notes

With the presence of the instructor, the learner can:

- Be taught those teaching materials that are difficult to present orally or with the use of the whiteboard and pen

- Be benefited by the oral explanation of the instructor plus the process visualization

- Ask the instructor to review some animation steps with losing much time. This is sometimes difficult or impossible to achieve in traditional teaching platforms

- Present her/his own doubts using the same tool used by the instructor

- Ask the instructor to improve the info-graphical explanation immediately by providing direct feedback. The changes are recorded in the platform and may be reviewed at a later stage

- Review the explanations and other learning materials in an integrated manner with the help of the instructor

- Answer evaluation tests using the same communication language and teaching platform 


\section{CONCLUSIONS AND FUTURE RESEARCH}

In this study, we reviewed teaching-learning and interaction paradigms with the aim of finding appropriate mechanisms for bi-directional communication between learner and instructor in an e-education environment. We selected animation as the visual language for achieving our purpose. Animation is appropriate for Internet-based communication since it does not need as much resources as video and sound, and it is easy to set by the instructor.

According to our literature review, cognitive load theory suggests that integrated instructional formats that are cognitively-designed reduce the learner's cognitive load, and therefore facilitate learning. We have designed a teaching-learning platform based on cognitive load theory, with the aim of reducing the extraneous cognitive load, and improving learning.

We built a prototype to represent our e-education teaching-learning platform. Our prototype was built in Java and can be used by any individual with Internet access. We have used our platform in networking and operating systems courses, and obtained a positive qualitative evaluation from the students. We are currently preparing a set of experiments to quantitatively evaluate our platform based on the amount and quality of learning achieved when this platform is used. In these experiments, we will set up a sequence of activities as follows:

- Pre-test

- Introduction to the platform

- Knowledge acquisition

- Practice with platform

- Testing

- Knowledge transfer evaluation

We will initially have groups of students that are taught by an instructor (present) and using the platform; taught through the platform in a distance learning situation; and taught by an instructor but without the platform.

The testing of acquired knowledge will provide an interesting evaluation of the use of our teaching-learning platform with and without the presence of the instructor in comparison to no using the platform at all. We will also register the level of difficulty of the presented materials as perceived by the learners during the knowledge acquisition phase (Pass et al., 1993). This will allow us to measure the imposed cognitive load and to test cognitive load theory.

Another important issue that we need to address is the learners' background knowledge problem. We need to investigate mechanisms for detecting the level of expertise of the learner, and to adapt the presentation format to this level. The specific objectives of our future research are summarized as follows: 
- Revision of cognitive load effects on the use of animation in an instructional format. This revision includes the experimental testing of the applicable cognitive load effects that were described above (i.e. worked-examples, split-attention, redundancy). The experimental work will differentiate the levels of interactivity of the materials presented to prove cognitive load theory.

- Revision of paradigms to evaluate learners' background knowledge. Application of interaction and evaluation paradigms to define a mechanism for presenting the appropriate level and format of instructional materials, especially for materials of high element interactivity.

- Implementation of a prototype representing the combined paradigms (mentioned above) that allow us to experiment and obtain results in terms of learners' performance and their perceived cognitive load. This prototype concentrates on the interaction between instructor and learner, specifically the evaluation of background knowledge and appropriate presentation of high element interactivity materials.

\section{REFERENCES}

Baddely, A. (1992). Working memory, Science, 255, 556-559.

Cerpa, N., Chandler P., \& Sweller, J. (1996). Some Conditions Under Which Integrated Computer-Based Training Software Can Facilitate Learning, Journal of Educational Computing Research, Volume 15, Number 4, 345-367.

Cooper, G. \& Sweller, J. (1987). The effects of schema acquisition and rule automation on mathematical problem-solving transfer. Journal of Educational Psychology, 79, 347-362.

Corry M. (1998). Mental models and hypermedia user interface design, AACE Educational Technology Review, Spring/Summer, 20-24

Chandler, P., \& Sweller, J. (1991). Cognitive load theory and the format of instruction. Cognition and Instruction, 8, 293-332.

Chandler, P., \& Sweller, J. (1992). The split attention effect as a factor in the design of instruction. British Journal of Educational Psychology, 62, 233-246.

Gick, M. L. \& Holyoak, K. J. (1983). Schema induction and analogical transfer. Cognitive Psychology, 15, 1-38.

Halford, G., Maybery, M., and Bain, J. (1986). Capacity limitations in children's reasoning; a dual task approach. Child Development, 57, 616-627.

Halford, G., Wilson, W., Guo, J., Wiles, J., and Stewart, J. (1994). Connectionist implications for processing capacity limitations in analogies. In J. Holyoak and J. Bamden (Eds.), Advances in connectionist and neural computation theory; Vol 2, Analogical connections, 363-415. Norwood, NJ: Ablex.

Halford, G., Wilson, W., Phillips, S. (1998). Processing capacity defined by relational complexity: Implications for comparative, developmental, and cognitive psychology. The Behavioral and Brain Sciences, 21(6), 803-831. 
Hegarty, M., Quilici, J., Narayanan, N.H., Holmquist, S., \& Moreno, R. (1999). Multimedia instruction: Lessons from evaluation of a theory-based design, Journal of Educational Multimedia and Hypermedia, 8, 119-150.

Holyoak, K. J. (1985). The pragmatics of analogical transfer. In G. H. Bower (Ed.), The psychology of learning and motivation, 9, 59-87. New York: Academic Press.

Kalyuga, S., Chandler, P., \& Sweller, J. (1998). Levels of expertise and instructional design. Human Factors, 40, 1-17.

Kalyuga S. (2000). When using sound with a text or picture is not beneficial for learning, Australian Journal of Educational Technology, 16(2), 161-172.

Kalyuga, S., Chandler, P., \& Sweller, J. (2000). Incorporating learner experience into the design of multimedia instruction. Journal of Educational Psychology, 92, 126-136.

Maybery, M., Bain, J., and Halford, G. (1986). Information processing demands of transitive inference. Journal of Experimental Psychology: Learning Memory and Cognition, 12, 600-613.

Najjar, L. (1996). Multimedia information and learning. Journal of Educational Multimedia and Hypermedia, 5, 129-150.

Pass, F., and Van Merrienboer, J. (1993). The efficiency of instructional conditions: an approach to combine mental effort and performance measures. Human Factors, 35(4), 737-743.

Pass, F., \& Van Merrienboer, J. (1994). Variability of worked examples and transfer of geometric problem-solving skills: A cognitive load approach. Journal of Educational Psychology, 86, 122-133.

Pavio, A. (1990). Mental representations: A dual-coding approach. New York :Oxford University Press.

Rumelhart, D. E. (1984). Schemata and the cognitive system. In R. S. Wyer and T. K. Scrull (Eds.), Handbook of social cognition, Vol 1, 161-188. Hillsdale, NJ; Erlbaum.

Rumelhart, D.E., and Norman, D. A. (1981). Analogical processes in learning. In J. R. Anderson (Ed.), Cognitive skills and their acquisition, 335-359, Hillsdale, NJ:Erlbaum.

Sweller, J. (1988). Cognitive load during problem solving: Effects on learning. Cognitive Science, 12, 257-285.

Sweller, J. (1989). Cognitive technology : some procedures for facilitating learning and problem solving in mathematics and science. Journal of Educational Psychology, 81, 457-466.

Sweller, J. (1991). Some modern myths of cognition and instruction. In J. B. Biggs (ed.), Teaching for Learning; The view from cognitive psychology: ACER, Radford House, Vic., Australia.

Sweller, J. (1993). Some cognitive processes and their consequences for the organisation and presentation of information. Australian Journal of Psychology, $45,1-8$.

Sweller, J. (1994). Cognitive load theory, learning difficulty, and instructional design. Learning and Instruction, 4, 295-312.

Sweller and Chandler, P. (1994). Why some material is difficult to learn. Cognition and Instruction, 12(3), 185-233.

Tergan, S. (1997). Misleading theoretical assumptions in hypertext/hypermedia research. Journal of Educational Multimedia and Hypermedia, 6, 257-283. 